\title{
Validation of maternal recall on exclusive breastfeeding 12 months after childbirth
}

\author{
BC Schneider ${ }^{1, *}$, BO Cata-Preta ${ }^{1}$, DD Gräf ${ }^{1}$, DLR Silva ${ }^{1}$, FS Santos ${ }^{1}$, MS Dias ${ }^{1}$,

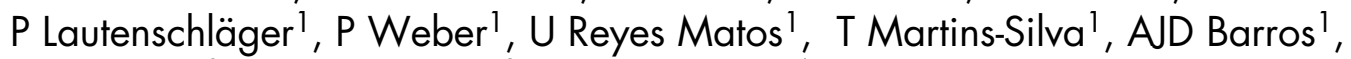 \\ FC Barros ${ }^{1,2}$, A Matijasevich ${ }^{3}$ and IS Santos ${ }^{1,4}$ \\ 'Postgraduate Program in Epidemiology, Universidade Federal de Pelotas, CEP 96020-220 Pelotas, RS, Brazil: \\ ${ }^{2}$ Postgraduate Course in Health and Behavior, Catholic University of Pelotas, Pelotas, RS, Brazil: ${ }^{3}$ Departamento de \\ Medicina Preventiva, Faculdade de Medicina FMUSP, Universidade de São Paulo, São Paulo, SP, Brazil: \\ 4Postgraduate Programme in Pediatrics and Child Health, Pontifical Catholic University of Rio Grande do Sul, Porto \\ Alegre, RS, Brazil
}

Submitted 30 July 2019: Final revision received 29 November 2019: Accepted 2 January 2020: First published online 27 May 2020

\begin{abstract}
Objective: We aimed to assess the validity of maternal recall of exclusive breastfeeding (EBF) at 3 months obtained 12 months after childbirth.

Design: A population-based birth cohort study. The gold standard is maternal report of $\mathrm{EBF}$ at the age of 3 months (yes or no) and age of introduction of other foods in the infant's diet. EBF was considered when the mother reported that no liquid, semi-solid or solid food was introduced up to that moment. The variable to be validated was obtained at 12 months after childbirth when the mother was asked about the age of food introduction. The prevalence of EBF at 3 months, and sensitivity, specificity, positive (PPV) and negative predictive values (NPV), and accuracy of 12-month recall with $95 \%$ CI were calculated.

Setting: Pelotas, Brazil.

Participants: 3700 mothers of participants of the Pelotas 2004 Birth Cohort.

Results: The prevalence of EBF at 3 months was $27 \cdot 8 \%$ (95\% CI 26.4, 29.3) and $49 \cdot 0 \%(95 \%$ CI $47 \cdot 4,50 \cdot 6)$ according to gold standard and maternal recall, respectively. The sensitivity of maternal recall at 12 months was 98.3\% (95\% CI 97.4, 99.0), specificity 70.0\% (95\% CI 68.2, 71.7), PPV 55.8\% (95\% CI 53.4, 58.1), NPV 99.1\% (95\% CI 98.6, 99.5) and accuracy 77.9\% (95\% CI 76.6. 79.2). When the analyses were stratified by maternal and infant characteristics, the sensitivity remained around $98 \%$, and the specificity ranged from 64.4 to $81.8 \%$. Conclusions: EBF recalled at the end of the first year of infant's life is a valid measure to be used in epidemiological investigations.
\end{abstract}

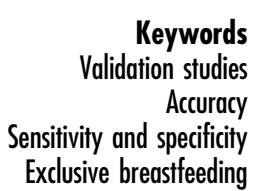

The WHO recommends exclusive breastfeeding (EBF) for the first 6 months of life ${ }^{(1)}$. The infant should not receive any liquid (water, teas or juices), semi-solid or solid food before reaching 6 months of age, except medicines and supplements ${ }^{(2)}$. In low- and middle-income countries, only $37 \%$ of infants $<6$ months of age are exclusively breastfed, and this prevalence is even lower in high-income countries ${ }^{(3)}$. Data from four national surveys collected through 24-h dietary recalls showed that EBF prevalence in Brazilian infants $<6$ months of age has increased from $2.9 \%$ in 1986 to $36.6 \%$ in 2013 , with a statistically significant increase in each decade up to 2006 and a posterior stabilisation until $2013^{(4)}$. Similarly, among infants $<2$ months of age, there was an increase of $44 \%$ in $\mathrm{EBF}$ prevalence between 1986 and 2006, and a reduction of $0.3 \%$ between 2006 and $2013^{(4)}$. In Pelotas, a city in southern Brazil, data from three population-based birth cohorts showed that the prevalence of EBF at 3 months of age increased from $7.0 \%$ in 1993 to $45.0 \%$ in $2015^{(5)}$.

Breastfeeding brings numerous benefits to the health of both mother and child ${ }^{(3,6-11)}$. The UNICEF and WHO recommend that countries should monitor breastfeeding rates at least every 5 years in order to detect negative trends and identify the need to direct resources for the strengthening of policies and programmes to promote breastfeeding ${ }^{(12)}$. Large national health surveys, such as the Multiple Indicator Cluster Survey (MICS) ${ }^{(13)}$ and the Demographic and Health Survey (DHS) ${ }^{(14)}$, which are responsible for 
collecting and disseminating accurate national nutrition and health data in low- and middle-income countries, as well as country-specific surveys, such as the National Health and Nutrition Survey (PNSN) ${ }^{(15)}$ in Brazil, are applied in the form of interviews, with the aid of structured questionnaires, with information about EBF usually obtained by the use of 24-h dietary recalls.

Despite the importance of the accuracy of information collected and the availability of studies that have assessed the validity of maternal recall of breastfeeding duration ${ }^{(16-18)}$, there are only few studies that have assessed the validity of maternal recall of duration of $\mathrm{EBF}^{(19-21)}$. Thus, the current study aimed to assess the validity of maternal information, obtained 12 months after childbirth, on the practice of EBF at 3 months of age among mothers of the Pelotas 2004 Birth Cohort in southern Brazil.

\section{Methods}

This study was conducted with data collected at birth and at 3- and 12-month follow-ups of the Pelotas 2004 Birth Cohort. All newborns in 2004, with at least $500 \mathrm{~g}$ of birthweight or 20 weeks of gestational age, whose mothers lived in the urban area of the municipality and in Jardim América, a neighbourhood adjacent to Pelotas belonging to a neighbouring municipality (Capão do Leão), were enrolled to the cohort. With a refusal rate of $0 \cdot 8 \%, 4231$ newborns from the five maternity hospitals in the city were included in the cohort. Follow-up rates at 3 and 12 months were 95.7 and $94.3 \%$, respectively. Further methodological details about the cohort are available in previous publications $^{(22,23)}$.

For the present investigation, only newborns of single births were considered. A total of 3700 mothers provided information about the patterns of breastfeeding at 3- and 12-month follow-ups. Maternal report on EBF obtained at 3 months of age was used as the gold standard for validation analysis. Initially, mothers were asked if the infant received breast milk. Then the mothers were questioned about the introduction of other foods by means of the following questions: 'When has <INFANT'S NAME > begun to eat regularly (being presented one by one the foods in the following list): cow's milk, powdered milk, tea, juice, water, mashed fruits, soup, mashed vegetables, porridge, egg and other food?'. Regular consumption was considered when the food had been offered at least twice, on different days, in the last week. The age of introduction of each food was recorded in months and days. EBF was recorded when breastfed children were not fed any other liquid, semi-solid or solid foods up to 3 months of age.

The variable to be validated was obtained at 12 months after childbirth when the mother was asked about the age of food introduction. The question used was: 'Now, I am going to tell you a list of liquids and foods and let me know if you have started giving them to <INFANT'S NAME $>$.
When I say "started", I want to know if <INFANT'S NAME $>$ receives or received that liquid or food every day, or almost every day of the week. If you have already started giving it, I want to know when you have started: cow's milk, powdered milk, tea, juice, mashed fruit, soup, mashed vegetables, porridge, yogurt, bread or cracker, egg (yolk), egg (white), meat, bean broth, bean grain, pasta, legumes/vegetables (in pieces), and other foods'. The age of food introduction was collected in months and days from the first ingestion of each food. When the mother reported that no other liquid, semi-solid or solid food but breastmilk was introduced up to the age of 3 months, the breastfeeding pattern at 3 months was recorded as EBF.

In order to characterise the sample, the following information about the infant at birth was used: gestational age (complete weeks), sex (male or female) and weight ( $<2500$ or $\geq 2500 \mathrm{~g}$ ). In addition, the following maternal characteristics were used: age at delivery (subsequently categorised as $\leq 19$, 20-29 or 30-46 years); self-reported skin colour (white, black or other); completed years of schooling at delivery (later categorised into 0-4, 5-8, 9-11 and $\geq 12$ years); socioeconomic level based on the criteria of the Research Companies Brazilian Association (ABEP), which uses schoolarity of the family head and household assets, categorised into economic classes A/B (wealthiest), $\mathrm{C}$ or $\mathrm{D} / \mathrm{E}$ (poorest) ${ }^{(24)}$; parity (number of children born alive or dead); maternal smoking during pregnancy (at least one cigarette per day in any trimester of gestation); marital status (without or with partner); self-reported depressive symptoms during pregnancy ('During pregnancy, did you have depression or nervous problem'? no; yes, treated; yes, untreated); type of delivery (vaginal or C-section); previous experience with breastfeeding (yes or no); and number of antenatal consultations. At the 3-month follow-up, the mothers were asked if they had returned to work after childbirth (yes or no) and if they were currently smokers (yes or no).

Statistical analyses were conducted in Stata, version 14.2 (StataCorp). To verify the sensitivity, specificity, positive (PPV) and negative predictive values (NPV), the statistical command 'diagt' was used. These parameters were first calculated for the entire sample and then after stratifying the sample according to the independent variables. Accuracy was calculated based on the following formula: (true positives + true negatives)/whole sample. The $95 \%$ CI was calculated for all the estimated parameters. True and false positive and negative values were defined as follows:

- True positives: mothers who reported EBF both at the 3-month (gold standard) and 12-month follow-ups;

- False negatives: mothers who reported EBF at the 3-month follow-up, but in the 12-month follow-up they reported having introduced any liquid, semisolid or solid food before the age of 3 months;

- True negatives: mothers not reporting EBF at the 3- and 12-month follow-ups; 
- False positives: mothers not reporting EBF at the 3-month follow-up as well as, at the 12-month follow-up, not having introduced any liquid, semisolid or solid food before the age of 3 months.

\section{Results}

At both follow-ups ( 3 and 12 months), the majority of mothers were between 20 and 29 years of age; self-reported white skin colour; lived with a partner; had two or more children; were non-smokers during or after pregnancy; had previous experience with breastfeeding; attended eight or more antenatal consultations; had a vaginal delivery; and had not returned to work after childbirth (Table 1). Approximately $20 \%$ of the mothers reported depressive symptoms during pregnancy, and most received no treatment for depression. The proportion of mothers with lower years of schooling ( $<9$ years) was higher at the 12 month (52.2\%) than at the 3-month follow-up (42.7\%), whereas the proportion from socioeconomic classes $\mathrm{D} / \mathrm{E}$ was higher at the 12 -month (44.3\%) than at the 3-month follow-up (38.6\%) (Table 1). As for infant characteristics (Table 1), the prevalence of low birthweight (LBW) $(<2500 \mathrm{~g})$ and preterm birth were 5.6 and $7.8 \%$, respectively, at the 3-month follow-up, and 9.5 and $12.6 \%$ at the 12-month follow-up.

The prevalence of EBF by maternal report at the 3-month follow-up (gold standard) was 27.8\% (95\% CI 26.4, 29.3). At the 12-month follow-up, the maternal report provided a prevalence of EBF at 3 months of $49.0 \%$ (95\% CI $47.4,50.6$ ) (data not presented in table). Maternal recall of EBF at 3 months at the end of the first year after childbirth showed a sensitivity of $98.3 \%$ (95\% CI 97.4, 99.0),

Table 1 Maternal and child characteristics of the Pelotas 2004 Birth Cohort at 3 and 12 months postpartum - Pelotas, Rio Grande do Sul, Brazil

\begin{tabular}{|c|c|c|c|c|c|c|}
\hline \multirow[b]{2}{*}{ Characteristics } & \multicolumn{2}{|c|}{ Sample*, $n 3700$} & \multicolumn{2}{|c|}{$\begin{array}{c}\text { EBF gold standard } \\
\text { (obtained at } 3 \text { months), } \\
n 1028\end{array}$} & \multicolumn{2}{|c|}{$\begin{array}{l}\text { EBF test (obtained at } \\
12 \text { months), } n 1813\end{array}$} \\
\hline & $n$ & $\%$ & $\%$ & $95 \% \mathrm{Cl}$ & $\%$ & $95 \% \mathrm{Cl}$ \\
\hline \multicolumn{7}{|l|}{ Maternal } \\
\hline Total & & & $27 \cdot 8$ & $26 \cdot 4,29 \cdot 3$ & $49 \cdot 0$ & $47 \cdot 4,50 \cdot 6$ \\
\hline \multicolumn{7}{|l|}{ Age (years) } \\
\hline$\leq 19$ & 681 & $18 \cdot 4$ & $12 \cdot 7$ & $10 \cdot 8,14 \cdot 8$ & $15 \cdot 6$ & $14 \cdot 0,17 \cdot 3$ \\
\hline $20-29$ & 1836 & $49 \cdot 6$ & $50 \cdot 6$ & $47 \cdot 6,53 \cdot 7$ & $50 \cdot 3$ & $48 \cdot 0,52 \cdot 6$ \\
\hline $30-46$ & 1182 & $32 \cdot 0$ & $36 \cdot 7$ & $33 \cdot 8,39 \cdot 7$ & 34.2 & $32 \cdot 0,36 \cdot 4$ \\
\hline \multicolumn{7}{|l|}{ Skin colour } \\
\hline White & 2716 & 73.4 & 74.6 & $77 \cdot 9,77 \cdot 2$ & $72 \cdot 8$ & $70 \cdot 7,74 \cdot 8$ \\
\hline Black & 731 & $19 \cdot 8$ & $18 \cdot 8$ & $16 \cdot 5,21 \cdot 3$ & $20 \cdot 5$ & $18 \cdot 7,22 \cdot 4$ \\
\hline Other & 253 & $6 \cdot 8$ & $6 \cdot 6$ & $5 \cdot 2,8 \cdot 3$ & $6 \cdot 7$ & $5.5,7.9$ \\
\hline \multicolumn{7}{|l|}{ Marital status } \\
\hline Without partner & 569 & $15 \cdot 4$ & $11 \cdot 0$ & $9 \cdot 2,13 \cdot 1$ & 14.5 & $12 \cdot 9,16 \cdot 3$ \\
\hline With partner & 3131 & 84.6 & 89.0 & $86 \cdot 9,90 \cdot 8$ & $85 \cdot 5$ & $83 \cdot 7,87 \cdot 1$ \\
\hline \multicolumn{7}{|c|}{ Schooling (complete years) } \\
\hline $0-4$ & 552 & $15 \cdot 1$ & $9 \cdot 6$ & $7 \cdot 9,11.5$ & 13.5 & $12 \cdot 0,15 \cdot 2$ \\
\hline $5-8$ & 1512 & $41 \cdot 2$ & 33.1 & $30 \cdot 2,36 \cdot 0$ & 38.7 & $36 \cdot 5,41 \cdot 0$ \\
\hline $9-11$ & 1236 & 33.7 & $42 \cdot 8$ & $38 \cdot 8,44 \cdot 8$ & $36 \cdot 0$ & $33 \cdot 8,38 \cdot 2$ \\
\hline \multirow{2}{*}{\multicolumn{7}{|c|}{ SES (ABEP) }} \\
\hline & & & & & & \\
\hline $\mathrm{D} / \mathrm{E}$ (poorest) & 1440 & 47.4 & 38.6 & $35.4,41.9$ & $44 \cdot 3$ & $41 \cdot 8,46 \cdot 8$ \\
\hline C & 1062 & 35.0 & $36 \cdot 8$ & $33 \cdot 7,40 \cdot 2$ & $36 \cdot 0$ & $33 \cdot 7,38.5$ \\
\hline A/B (wealthiest) & 536 & $17 \cdot 6$ & 24.5 & $21 \cdot 7,27 \cdot 5$ & $19 \cdot 6$ & $17 \cdot 7,21 \cdot 7$ \\
\hline \multicolumn{7}{|l|}{ Parity } \\
\hline 1 & 1448 & 39.1 & $36 \cdot 4$ & $33 \cdot 5,39 \cdot 4$ & 37.7 & $35.4,39.9$ \\
\hline$\geq 2$ & 2252 & $60 \cdot 9$ & 63.6 & $60 \cdot 6,66 \cdot 5$ & $62 \cdot 3$ & $60 \cdot 0,64 \cdot 5$ \\
\hline \multicolumn{7}{|c|}{ Smoking during pregnancy } \\
\hline No & 2736 & 73.9 & 81.4 & $78 \cdot 9,83 \cdot 7$ & $76 \cdot 5$ & $74.5,78.4$ \\
\hline Yes & 964 & $26 \cdot 1$ & $18 \cdot 6$ & $16 \cdot 3,21 \cdot 0$ & $23 \cdot 5$ & $21 \cdot 6,25 \cdot 5$ \\
\hline \multicolumn{7}{|c|}{ Maternal smoking at 3 months postpartum } \\
\hline No & 2764 & 74.7 & $82 \cdot 4$ & $79 \cdot 9,84,6$ & 77.7 & $75 \cdot 7,79 \cdot 5$ \\
\hline Yes & 936 & $25 \cdot 3$ & $17 \cdot 6$ & $15 \cdot 4,20 \cdot 1$ & $22 \cdot 3$ & $20 \cdot 5,24 \cdot 3$ \\
\hline \multicolumn{7}{|c|}{ Depressive symptoms during pregnancy } \\
\hline No & 2783 & $75 \cdot 2$ & 78.4 & $75 \cdot 9,80 \cdot 9$ & $77 \cdot 7$ & $75 \cdot 7,79 \cdot 6$ \\
\hline Yes, untreated & 795 & 21.5 & $18 \cdot 3$ & $16 \cdot 1,20 \cdot 8$ & $19 \cdot 6$ & $17 \cdot 8,21 \cdot 5$ \\
\hline Yes, treated & 121 & $3 \cdot 3$ & 3.2 & $2 \cdot 3,4.5$ & $2 \cdot 7$ & $2 \cdot 0,3 \cdot 6$ \\
\hline \multicolumn{7}{|c|}{ Previous breastfeeding experience } \\
\hline No & 1809 & 48.9 & 43.7 & $40 \cdot 7,46 \cdot 7$ & $45 \cdot 7$ & $43 \cdot 4,48 \cdot 0$ \\
\hline Yes & 1891 & $51 \cdot 1$ & $56 \cdot 3$ & $53 \cdot 3,59 \cdot 3$ & $54 \cdot 3$ & $52 \cdot 0,56 \cdot 6$ \\
\hline
\end{tabular}


Table 1 Continued

\begin{tabular}{|c|c|c|c|c|c|c|}
\hline \multirow[b]{2}{*}{ Characteristics } & \multicolumn{2}{|c|}{ Sample* $^{*} n 3700$} & \multicolumn{2}{|c|}{$\begin{array}{c}\text { EBF gold standard } \\
\text { (obtained at } 3 \text { months), } \\
n 1028\end{array}$} & \multicolumn{2}{|c|}{$\begin{array}{l}\text { EBF test (obtained at } \\
12 \text { months), } n 1813\end{array}$} \\
\hline & $n$ & $\%$ & $\%$ & $95 \% \mathrm{Cl}$ & $\%$ & $95 \% \mathrm{Cl}$ \\
\hline \multicolumn{7}{|c|}{ Type of delivery } \\
\hline Vaginal & 2020 & 54.6 & 53.7 & $50 \cdot 6,56 \cdot 7$ & $54 \cdot 7$ & $52 \cdot 5,57 \cdot 1$ \\
\hline C-section & 1680 & $45 \cdot 4$ & $46 \cdot 3$ & $43 \cdot 3,49 \cdot 4$ & $45 \cdot 2$ & $42 \cdot 9,47 \cdot 5$ \\
\hline \multicolumn{7}{|c|}{ Number of antenatal consultations } \\
\hline$<8$ & 1413 & $39 \cdot 8$ & 31.4 & $28 \cdot 6,34 \cdot 4$ & $37 \cdot 6$ & $35.4,39.9$ \\
\hline$\geq 8$ & 2137 & $60 \cdot 2$ & $68 \cdot 6$ & $65 \cdot 6,71.4$ & $62 \cdot 4$ & $60 \cdot 1,64 \cdot 6$ \\
\hline \multicolumn{7}{|c|}{ Maternal postpartum work } \\
\hline No & 3209 & $86 \cdot 7$ & $89 \cdot 0$ & $86 \cdot 9,90 \cdot 8$ & 87.9 & $86 \cdot 3,89 \cdot 3$ \\
\hline Yes & 491 & 13.3 & 11.0 & $9 \cdot 2,13 \cdot 1$ & $12 \cdot 1$ & $10 \cdot 7,13 \cdot 7$ \\
\hline \multicolumn{7}{|l|}{ Infant } \\
\hline \multicolumn{7}{|l|}{ Sex } \\
\hline Male & 1911 & $51 \cdot 7$ & $50 \cdot 0$ & $46 \cdot 9,53 \cdot 1$ & $50 \cdot 3$ & $48 \cdot 0,52 \cdot 6$ \\
\hline Female & 1789 & $48 \cdot 4$ & $50 \cdot 0$ & $46 \cdot 9,53 \cdot 1$ & $49 \cdot 7$ & $47 \cdot 3,52 \cdot 0$ \\
\hline \multicolumn{7}{|c|}{ Birth weight $(\mathrm{g})$} \\
\hline$<2500$ & 322 & 8.7 & $5 \cdot 6$ & $6 \cdot 4,9 \cdot 6$ & $7 \cdot 8$ & $6 \cdot 6,9 \cdot 1$ \\
\hline$\geq 2500$ & 3377 & $91 \cdot 3$ & 94.4 & $92 \cdot 8,95 \cdot 6$ & $92 \cdot 2$ & $90 \cdot 9,93.4$ \\
\hline \multicolumn{7}{|c|}{ Gestational age (weeks) } \\
\hline$\leq 36$ & 499 & $13 \cdot 5$ & 9.5 & $7 \cdot 9,11 \cdot 5$ & $12 \cdot 6$ & $11 \cdot 2,14 \cdot 2$ \\
\hline$\geq 37$ & 3197 & $86 \cdot 5$ & $90 \cdot 5$ & $88 \cdot 5,92 \cdot 1$ & $87 \cdot 4$ & $85 \cdot 8,88 \cdot 8$ \\
\hline
\end{tabular}

EBF, exclusive breastfeeding; SES, socioeconomic status; ABEP, Research Companies Brazilian Association.

${ }^{*}$ Mothers who reported about exclusive breastfeeding at 3 and 12 months postpartum.

Table 2 Validity of maternal recall on exclusive breastfeeding (EBF) at 3 months, obtained at 12 months after childbirth, compared with the gold standard (EBF at 3 months, obtained at 3-month follow-up) - Pelotas 2004 Birth Cohort, Rio Grande do Sul, Brazil

\begin{tabular}{lcccc}
\hline Parameters & $n$ & $N$ & $\%$ & $95 \% \mathrm{Cl}$ \\
\hline Sensitivity & 1011 & 1028 & $98 \cdot 3$ & $97 \cdot 4,99 \cdot 0$ \\
Specificity & 1870 & 2672 & $70 \cdot 0$ & $68 \cdot 2,71 \cdot 7$ \\
PPV $^{*}$ & 1011 & 1813 & $55 \cdot 8$ & $53 \cdot 4,58 \cdot 1$ \\
NPV $^{*}$ & 1870 & 1887 & $99 \cdot 1$ & $98 \cdot 6,99 \cdot 5$ \\
Accuracy & $1011+1870$ & 3700 & $77 \cdot 9$ & $76 \cdot 6,79 \cdot 2$
\end{tabular}

PPV, positive predictive value; NPV, negative predictive value.

${ }^{*} P P V$ and NPV at an EBF prevalence of $27.8 \%$.

specificity of $70.0 \%(95 \%$ CI $68.2,71.7)$ and accuracy of $77.9 \%(95 \%$ CI 76.6, 79.2). PPV and NPV were $55.8 \%$ (95\% CI 53.4, 58.1) and 99.1\% (95\% CI 98.6, 99.5), respectively (Table 2).

The parameters of validation estimated according to maternal and newborn characteristics are described in Table 3. Among the different variables, sensitivity remained nearly $98.0 \%$, while specificity ranged from 66.4 to $81.8 \%$. PPV was higher among non-adolescent mothers, those who lived with a partner (58.1\%), those with $\geq 12$ years of formal education $(74.4 \%)$, those who belonged to socioeconomic level A/B (69.4\%), those who did not smoke during pregnancy $(59.4 \%)$ or at the 3-month follow-up (59.3\%), and those who attended eight or more antenatal care consultations (61.6\%), as well as among mothers of infants with birth weight $\geq 2500 \mathrm{~g}$ (57.0\%) or $\geq 37$ weeks of gestational age (57.7\%).

\section{Discussion}

The present study tested the validity of maternal recall on EBF at 3 months of age obtained 12 months after childbirth. The probability of maternal recall at the end of first year of child's life correctly identifying mothers who reported EBF at 3 months after delivery was $98.3 \%$. The specificity at 12 months was lower, with a $30 \%$ rate of false-positive responses, possibly indicating that mothers - despite being aware that EBF is advocated up to the first 6 months of infant's life - reported having offered it even when they did not. The accuracy of almost $80 \%$ is less than perfect or substantial but can be considered moderate ${ }^{(25)}$, indicating that maternal recall on EBF at 3 months of age collected after 12 months of childbirth is a valid measure deserving application in surveillance studies.

The measurement of EBF is complex, as rates may vary according to the definition, measurement period, instrument of assessment and even child's age ${ }^{(26)}$. Although maternal recall on breastfeeding has been widely used in research, its validity and reliability have been questioned, because it is a memory-dependent information ${ }^{(18)}$. However, a literature review of articles published between 1966 and 2003 in English has found that maternal report on initiation and duration of any breastfeeding would be accurate and reliable, although the recall of breastfeeding duration might become less accurate as the period of recall increases ${ }^{(16)}$. On the other hand, the validity and reliability of maternal recall of EBF duration might be less accurate ${ }^{(16)}$. 
Table 3 Validation of maternal recall on exclusive breastfeeding at 3 months, obtained at 12 months after childbirth, according to maternal and infant characteristics - Pelotas 2004 Birth Cohort, Rio Grande do Sul, Brazil ( $n$ 3700)

\begin{tabular}{|c|c|c|c|c|c|c|c|c|}
\hline \multirow[b]{2}{*}{ Characteristics } & \multicolumn{2}{|c|}{ Sensitivity } & \multicolumn{2}{|c|}{ Specificity } & \multicolumn{2}{|c|}{ PPV } & \multicolumn{2}{|c|}{ NPV } \\
\hline & $\%$ & $95 \% \mathrm{Cl}$ & $\%$ & $95 \% \mathrm{Cl}$ & $\%$ & $95 \% \mathrm{Cl}$ & $\%$ & $95 \% \mathrm{Cl}$ \\
\hline \multicolumn{9}{|c|}{ Maternal } \\
\hline \multicolumn{9}{|c|}{ Age (complete years) } \\
\hline$\leq 19$ & 98.5 & $94 \cdot 6,99 \cdot 8$ & $72 \cdot 1$ & $68 \cdot 1,75 \cdot 8$ & 45.4 & $39.5,51.4$ & 99.5 & $98.2,99.9$ \\
\hline $20-29$ & $98 \cdot 3$ & $96 \cdot 7,99 \cdot 2$ & $69 \cdot 6$ & $67 \cdot 0,72 \cdot 1$ & $56 \cdot 1$ & $52 \cdot 8,59 \cdot 3$ & 99.0 & $98 \cdot 2,99 \cdot 6$ \\
\hline $30-46$ & $98 \cdot 4$ & $96 \cdot 2,99 \cdot 4$ & 69.2 & $65 \cdot 9,72 \cdot 4$ & 59.9 & $56 \cdot 0,63 \cdot 8$ & 98.9 & $97 \cdot 7,99 \cdot 6$ \\
\hline \multicolumn{9}{|l|}{ Skin colour } \\
\hline White & 98.0 & $96 \cdot 8,98 \cdot 9$ & 70.9 & $68.8,72 \cdot 9$ & $57 \cdot 0$ & $54 \cdot 2,59 \cdot 7$ & 98.9 & $98 \cdot 2,99.4$ \\
\hline Black & 99.0 & $96 \cdot 3,99 \cdot 9$ & 66.5 & $62.4,70.5$ & 51.5 & $46 \cdot 3,56 \cdot 7$ & $99 \cdot 4$ & $98 \cdot 0,99 \cdot 9$ \\
\hline Other & 100 & $94.7,100$ & $70 \cdot 8$ & $63 \cdot 7,77 \cdot 2$ & 55.7 & $46 \cdot 5,64 \cdot 7$ & 100 & $97.2,100$ \\
\hline \multicolumn{9}{|l|}{ Marital status } \\
\hline Without partner & $97 \cdot 3$ & $92 \cdot 4,99 \cdot 4$ & 66.4 & $61.9,70 \cdot 8$ & $41 \cdot 8$ & $35 \cdot 8,48 \cdot 0$ & 99.0 & $97 \cdot 2,99 \cdot 8$ \\
\hline With partner & 98.5 & $97 \cdot 4,99 \cdot 2$ & $70 \cdot 7$ & $68 \cdot 8,72 \cdot 6$ & $58 \cdot 1$ & $55 \cdot 6,60 \cdot 6$ & $99 \cdot 1$ & $98.5,99.5$ \\
\hline \multicolumn{9}{|c|}{ Schooling (complete years) } \\
\hline $0-4$ & 99.0 & $94.4,100$ & $67 \cdot 7$ & $63 \cdot 2,72 \cdot 0$ & 39.5 & $33 \cdot 3,46 \cdot 0$ & 99.7 & $98 \cdot 2,100$ \\
\hline $5-8$ & 98.5 & $96.6,99.5$ & $69 \cdot 0$ & $66 \cdot 3,71 \cdot 6$ & 47.5 & $43 \cdot 7,51 \cdot 3$ & 99.4 & $98 \cdot 6,99 \cdot 8$ \\
\hline $9-11$ & 97.6 & $95 \cdot 7,98.9$ & $71 \cdot 3$ & $68 \cdot 1,74 \cdot 4$ & 63.9 & $60 \cdot 1,67 \cdot 6$ & $98 \cdot 3$ & $96 \cdot 9,99 \cdot 2$ \\
\hline$\geq 12$ & $99 \cdot 4$ & $96 \cdot 5,100$ & $74 \cdot 0$ & $67.5,79.9$ & 74.4 & $68 \cdot 0,80 \cdot 2$ & 99.4 & $96.5,100$ \\
\hline \multicolumn{9}{|l|}{ SES (ABEP) } \\
\hline $\mathrm{D} / \mathrm{E}$ (poorest) & $98 \cdot 2$ & $96 \cdot 1,99 \cdot 3$ & $69 \cdot 0$ & $66 \cdot 2,71 \cdot 7$ & $48 \cdot 8$ & $45 \cdot 0,52 \cdot 7$ & 99.2 & $98 \cdot 3,99 \cdot 7$ \\
\hline C & 99.4 & $97 \cdot 7,99 \cdot 9$ & $69 \cdot 2$ & $65 \cdot 8,72 \cdot 5$ & $58 \cdot 0$ & $53 \cdot 7,62 \cdot 2$ & $99 . \overline{6}$ & $98.6,100$ \\
\hline $\mathrm{A} / \mathrm{B}$ (wealthiest) & 97.6 & $94.6,99.2$ & 72.0 & $66 \cdot 8,76 \cdot 8$ & 69.4 & $63 \cdot 8,74 \cdot 6$ & 97.9 & $95 \cdot 2,99 \cdot 3$ \\
\hline \multicolumn{9}{|l|}{ Parity } \\
\hline 1 & 97.9 & $95 \cdot 8,99 \cdot 1$ & 70.5 & $67 \cdot 7,73 \cdot 2$ & $53 \cdot 6$ & $49 \cdot 8,57 \cdot 4$ & 99.0 & $97.9,99.5$ \\
\hline$\geq 2$ & 98.6 & $97 \cdot 4,99 \cdot 4$ & $69 \cdot 6$ & $67.3,71.9$ & $57 \cdot 1$ & $54 \cdot 1,60 \cdot 0$ & 99.2 & $98 \cdot 5,99 \cdot 6$ \\
\hline \multicolumn{9}{|c|}{ Smoking during pregnancy } \\
\hline No & 98.4 & $97.4,99.2$ & 70.4 & $68 \cdot 2,72 \cdot 4$ & 59.4 & $56 \cdot 8,62 \cdot 0$ & 99.0 & $98 \cdot 4,99.5$ \\
\hline Yes & 97.9 & $94.7,99 \cdot 4$ & $69 \cdot 1$ & $65 \cdot 7,72 \cdot 3$ & 43.9 & $39 \cdot 1,48 \cdot 8$ & $99 \cdot 3$ & $98 \cdot 1,99.8$ \\
\hline \multicolumn{9}{|c|}{ Maternal smoking at 3 months postpartum } \\
\hline No & 98.5 & $97.4,99.2$ & $70 \cdot 1$ & $68 \cdot 0,72 \cdot 1$ & $59 \cdot 2$ & $56 \cdot 6,61 \cdot 8$ & 99.0 & $98.4,99.5$ \\
\hline Yes & $97 \cdot 8$ & $94.4,99.4$ & $69 \cdot 8$ & $66 \cdot 4,73 \cdot 1$ & 43.7 & $38 \cdot 8,48 \cdot 7$ & $99 \cdot 2$ & $98 \cdot 1,99 \cdot 8$ \\
\hline \multicolumn{9}{|c|}{ Depressive symptoms during pregnancy } \\
\hline No & 98.4 & $97 \cdot 3,99 \cdot 1$ & 68.9 & $66 \cdot 8,70 \cdot 9$ & $56 \cdot 3$ & $53 \cdot 7,58 \cdot 9$ & $99 \cdot 1$ & $98.4,99.5$ \\
\hline Yes, untreated & 97.9 & $94.6,99 \cdot 4$ & 71.8 & $68 \cdot 1,75 \cdot 4$ & $51 \cdot 8$ & $46 \cdot 5,57 \cdot 1$ & $99 \cdot 1$ & $97 \cdot 7,99 \cdot 8$ \\
\hline Yes, treated & 100 & $89 \cdot 4,100$ & $81 \cdot 8$ & $72 \cdot 2,89 \cdot 2$ & $67 \cdot 3$ & $52 \cdot 5,80 \cdot 1$ & 100 & $95 \cdot 0,100$ \\
\hline \multicolumn{9}{|c|}{ Previous breastfeeding experience } \\
\hline No & $98 \cdot 0$ & $96 \cdot 2,99 \cdot 1$ & 71.4 & $68 \cdot 9,73 \cdot 8$ & $53 \cdot 1$ & $49 \cdot 6,56 \cdot 1$ & 99.1 & $98 \cdot 3,99 \cdot 6$ \\
\hline Yes & 98.6 & $97 \cdot 3,99 \cdot 4$ & 68.5 & $65.9,71.0$ & 58.0 & $54 \cdot 9,61 \cdot 1$ & 99.1 & $98 \cdot 3,99 \cdot 6$ \\
\hline Type of delivery & & & & & & & & \\
\hline Vaginal & 98.4 & $96 \cdot 9,99 \cdot 3$ & $69 \cdot 3$ & $66 \cdot 9,71 \cdot 7$ & $54 \cdot 7$ & $51 \cdot 5,57 \cdot 8$ & 99.1 & $98 \cdot 3,99 \cdot 6$ \\
\hline C-section & $98 \cdot 3$ & $96 \cdot 7,99 \cdot 3$ & $70 \cdot 8$ & $68 \cdot 1,73 \cdot 3$ & $57 \cdot 1$ & $53 \cdot 6,60.5$ & 99.1 & $98 \cdot 2,99 \cdot 6$ \\
\hline Number of antenat & & & & & & & & \\
\hline$<8$ & $97 \cdot 7$ & $95.4,99.1$ & $68 \cdot 1$ & $65 \cdot 3,70 \cdot 9$ & 46.4 & $42 \cdot 5,50 \cdot 3$ & 99.1 & $98 \cdot 1,99.6$ \\
\hline$\geq 8$ & $98 \cdot 8$ & $97.7,99.5$ & 71.4 & $69 \cdot 0,73 \cdot 7$ & $61 \cdot 6$ & $58 \cdot 7,64.5$ & $99 \cdot 2$ & $98 \cdot 5,99 \cdot 7$ \\
\hline Maternal postpartu & & & & & & & & \\
\hline No & $98 \cdot 3$ & $97 \cdot 2,99 \cdot 0$ & $69 \cdot 7$ & $67 \cdot 8,71 \cdot 6$ & $56 \cdot 4$ & $54 \cdot 0,58 \cdot 9$ & 99.0 & $98 \cdot 4,99 \cdot 4$ \\
\hline Yes & $99 \cdot 1$ & $95 \cdot 2,100$ & 71.4 & $66 \cdot 6,75 \cdot 9$ & $50 \cdot 9$ & $44 \cdot 1,57 \cdot 7$ & $99 \cdot 6$ & $98.0,100$ \\
\hline Infant & & & & & & & & \\
\hline Sex & & & & & & & & \\
\hline Male & $98 \cdot 6$ & $97 \cdot 2,99 \cdot 5$ & 71.0 & $68.6,73.4$ & $55 \cdot 6$ & $52 \cdot 3,58 \cdot 8$ & $99 \cdot 3$ & $98 \cdot 6,99 \cdot 7$ \\
\hline Female & $98 \cdot 1$ & $96 \cdot 5,99 \cdot 1$ & 68.9 & $66 \cdot 2,71 \cdot 4$ & 55.9 & $52 \cdot 6,59 \cdot 2$ & 98.9 & $97.9,99.5$ \\
\hline Birth weight $(\mathrm{g})$ & & & & & & & & \\
\hline$<2500$ & 100 & $93 \cdot 8,100$ & 68.6 & $62 \cdot 6,74 \cdot 1$ & $41 \cdot 1$ & $32 \cdot 9,49 \cdot 7$ & 100 & $98.0,100$ \\
\hline$\geq 2500$ & $98 \cdot 2$ & $97 \cdot 2,99 \cdot 0$ & $70 \cdot 1$ & $68 \cdot 3,72 \cdot 0$ & $57 \cdot 0$ & $54 \cdot 6,59 \cdot 4$ & 99.0 & $98 \cdot 4,99 \cdot 4$ \\
\hline Gestational age (w & & & & & & & & \\
\hline$\leq 36$ & 99.0 & $94 \cdot 4,100$ & $67 \cdot 1$ & $62 \cdot 2,71 \cdot 7$ & $42 \cdot 4$ & $35 \cdot 9,49 \cdot 0$ & 99.6 & $98 \cdot 0,100$ \\
\hline$\geq 37$ & $98 \cdot 3$ & $97 \cdot 2,99 \cdot 0$ & 70.5 & $68 \cdot 6,72 \cdot 4$ & $57 \cdot 7$ & $55 \cdot 3,60 \cdot 2$ & 99.0 & $98.4,99 \cdot 4$ \\
\hline
\end{tabular}

PPV, positive predictive value; NPV, negative predictive value; SES, socioeconomic status; ABEP, Research Companies Brazilian Association.

More recently, a survey conducted in the United States has shown that maternal recall on any breastfeeding duration in the first year of child's life verified 6 years after childbirth had a high intraclass correlation coefficient
$($ ICC $=0.84)$ against the gold standard obtained monthly by maternal report until 12 months of age ${ }^{(17)}$. In Brazil, researchers have found a high agreement $(I C C=0.92)$ between maternal recall on any breastfeeding duration 
collected when the child was 2 years of age compared with reports collected monthly in the first 3 months of child's life (gold standard) ${ }^{(18)}$, which is consistent with our findings.

In terms of EBF, an investigation has found that at 2 years after delivery, mothers overestimated EBF up to 6 months of age as compared with the gold standard measured weekly up to 1 month of life and monthly up to the age of 6 months ${ }^{(19)}$. A study in Guatemala tested two selfreported instruments to assess EBF in infants aged 3 months using the dose-to-mother deuterium oxide turnover (DMDOT) technique as the reference method. The prevalence of EBF was $50 \%$ according to the current feeding practice reported, $61 \%$ by the $24-\mathrm{h}$ dietary recall and only $36 \%$ when using DMDOT. The sensitivity to detect EBF from the mother's report was $92 \%$ (95\% CI 62, 99), but from the 24-h dietary recall was $100 \%$ (95\% CI 72, 100). The specificity for both methods was lower - 74\% (95\% CI 51,89$)$ for reported current feeding practice and $61 \%$ (95\% CI 39, 79) for the 24-h dietary recall ${ }^{(21)}$.

In Sri Lanka, two methods to collect retrospective data on EBF up to 6 months of age were tested: (1) based on an event calendar (date of introduction, frequency of use and quantity of specific food items) and (2) mother's recall at 9 months after childbirth ${ }^{(20)}$. The gold standard was obtained through prospective data collected since birth. The authors have reported $100 \%$ sensitivity for both methods, specificity of $26 \cdot 2 \%$ (95\% CI $17 \cdot 9,36 \cdot 8)$ for an event calendar, and specificity of $75.0 \%$ (95\% CI 64.5, 83.2) for mother's recall at 9 months after childbirth ${ }^{(20)}$. The way the questions are framed may affect the accuracy of response from the mother. Asking the time of complementary feeding introduction (as in our study) may provide different results than asking the duration of EBF.

Some studies have reported differences in the validity of maternal recall on breastfeeding according to sociodemographic characteristics ${ }^{(17,19)}$. In our study, however, the sensitivity remained stable and around $98 \%$ at the different maternal and child characteristics evaluated. On the contrary, PPV varied according to maternal age, economic class, schooling, smoking, number of antenatal care consultations attended, birthweight and gestational age, thus reflecting differences in the prevalence of EBF among these groups of mothers. Youngest mothers, those under socioeconomically disadvantaged conditions as well as smokers presented the lowest rates of EBF at 3 months. Such findings are in agreement with the results of Amissah et al. from the USA ${ }^{(17)}$.

Our study has few strengths and limitations. The main strength is it being a population-based study. In addition, the gold standard information was collected near the moment of its occurrence. The mean age of infants when the follow-up occurred was 3.0 (SD 0.1) months, thus reducing the probability of recall bias. Among the limitations, it is possible that mothers taking part in a birth cohort study tend to remember more promptly of the events that occurred in the child's life than non-participating mothers, thus compromising the external validity of our results. Another point to consider is the information bias arising from the maternal knowledge about the recommendation of EBF for the first 6 months of infant's life. As a result, false-positive responses may be present in our findings at both follow-ups, thus increasing the estimated prevalence of EBF at 3 months and decreasing the specificity of maternal recall at 12 months.

\section{Conclusion}

This study contributes valuable data to epidemiological research on maternal recall of $\mathrm{EBF}$. Information relating to EBF at 3 months of age obtained from mothers 12 months after childbirth showed almost $80 \%$ accuracy. The information remained valid even after the sample was stratified by newborn weight, gestational age and several maternal characteristics.

\section{Acknowledgements}

Acknowledgements: This article is based on the data reported by the study 'Pelotas 2004 Birth Cohort' conducted by Postgraduate Programme in Epidemiology at Universidade Federal de Pelotas, with the collaboration of the Brazilian Public Health Association (ABRASCO). The WHO, the National Support Program for Centers of Excellence (PRONEX), the Brazilian National Research Council (CNPq), the Brazilian Ministry of Health and the Children's Mission supported the 2004 Birth Cohort study. Financial support: This study was financed in part by the Coordenação de Aperfeiçoamento de Pessoal de Nível Superior - Brazil (CAPES), finance code 001. A.M., A.J.D.B. and I.S.S. are supported by CNPq. Conflict of interest: The authors have no conflicts of interest directly relevant to the content of this article. Authorship: I.S.S., A.M., T.M.S. and B.C.S. conceived and designed the study. B.C.S., B.O.C.P., D.D.G., F.S.S., M.D.D., P.L., P.W. and U.M.R. analysed, interpreted the data and drafted the manuscript. I.S.S., A.M., A.J.D.B., F.C.B. and T.M.S. critically revised the manuscript. All authors approved the final version of the manuscript for submission. Ethics of buman subject participation: This study was conducted according to the guidelines laid down in the Declaration of Helsinki, and all procedures involving study participants were approved by the Research Ethics Committee of the Faculty of Medicine of the Federal University of Pelotas. All interviews were performed only after obtaining written informed consent from participating mothers. 


\section{References}

1. World Health Organization (2003) Complementary feeding: report of the global consultation, and summary of guiding principles for complementary feeding of the breastfed child. World Health Organization. https://apps.who.int/iris/ handle/10665/42739 (accessed November 2019).

2. World Health Organization (2008) Indicators for assessing infant and young child feeding practices: part 1: definitions: conclusions of a consensus meeting held 6-8 November 2007 in Washington DC, USA.

3. Victora CG, Bahl R, Barros AJ et al. (2016) Breastfeeding in the 21st century: epidemiology, mechanisms, and lifelong effect. Lancet 387, 475-490.

4. Boccolini CS, Boccolini PMM, Monteiro FR et al. (2017) Tendência de indicadores do aleitamento materno no Brasil em três décadas [Breastfeeding indicators trends in Brazil for three decades]. Revista de Saúde Pública 51, 1-9.

5. Santos IS, Barros FC, Horta BL et al. (2019) Breastfeeding exclusivity and duration: trends and inequalities in four population-based birth cohorts in Pelotas, Brazil, 1982-2015. Int J Epidemiol 48, i72-i79.

6. Victora CG, Horta BL, Loret de Mola C et al. (2015) Association between breastfeeding and intelligence, educational attainment, and income at 30 years of age: a prospective birth cohort study from Brazil. Lancet Glob Health 3, e199-e205.

7. Comite de nutrition de la Societe francaise de p; Turck D, Vidailhet M, Bocquet A et al. (2013) Breastfeeding: health benefits for child and mother. Arch Pediatr 20, Suppl. 2, S29-S48.

8. Horta BL (2019) Breastfeeding: investing in the future. Breastfeed Med 14, S11-S12.

9. Chowdhury R, Sinha B, Sankar MJ et al. (2015) Breastfeeding and maternal health outcomes: a systematic review and meta-analysis. Acta Paediatr 104, 96-113.

10. Sankar MJ, Sinha B, Chowdhury R et al. (2015) Optimal breastfeeding practices and infant and child mortality: a systematic review and meta-analysis. Acta Paediatr 104, 3-13.

11. Marseglia L, Manti S, D'Angelo G et al. (2015) Obesity and breastfeeding: the strength of association. Women Birth 28, 81-86.

12. UNICEF | World Health Organization (2017) Global Breastfeeding Scorecard: Tracking Breastfeeding Policies and Programmes. https://www.who.int/nutrition/publications/ infantfeeding/global-bf-scorecard-2017.pdf?ua=1 (accessed November 2019).
13. UNICEF | World Health Organization. Multiple Indicator Cluster Survey (MICS). https://www.unicef.org/statistics/ index_24302.html (accessed November 2019).

14. USAID | Demographic and Health Survey. https:// globalnutritionreport.org/reports/2016-global-nutrition-report/ (accessed November 2019).

15. Brasil Ministério da Saúde. Pesquisa Nacional de Alimentação e Nutrição [Brazil. Ministry of Health. National Food and Nutrition Policy (PNAN)]. http://aps.saude.gov.br (accessed November 2019).

16. Li R, Scanlon KS \& Serdula MK (2005) The validity and reliability of maternal recall of breastfeeding practice. Nutr Rev 63, 103-110.

17. Amissah EA, Kancherla V, Ko YA et al. (2017) Validation study of maternal recall on breastfeeding duration 6 years after childbirth. J Hum Lact 33, 390-400.

18. Barbosa RW, Oliveira AE, Zandonade E et al. (2012) Mothers' memory about breastfeeding and sucking habits in the first months of life for their children. Revista Paulista de Pediatria 30, 180-186.

19. Burnham L, Buczek M, Braun N et al. (2014) Determining length of breastfeeding exclusivity: validity of maternal report 2 years after birth. J Hum Lact 30, 190-194.

20. Agampodi SB, Fernando S, Dharmaratne SD et al. (2011) Duration of exclusive breastfeeding; validity of retrospective assessment at nine months of age. BMC Pediatr 11, 80.

21. Mazariegos M, Slater C \& Ramirez-Zea M (2016) Validity of Guatemalan mother's self-reported breast-feeding practices of 3-month-old infants. Food Nutr Bull 37, 494-503.

22. Santos IS, Barros AJ, Matijasevich A et al. (2011) Cohort profile: the 2004 Pelotas (Brazil) birth cohort study. Int $J$ Epidemiol 40, 1461-1468.

23. Santos IS, Barros AJ, Matijasevich A et al. (2014) Cohort profile update: 2004 Pelotas (Brazil) Birth Cohort Study. Body composition, mental health and genetic assessment at the 6 years follow-up. Int J Epidemiol 43, 1437-1437f.

24. Associação Brasileira de Empresas de Pesquisa [Brazilian Association of Research Companies (ABEP)]. http://www. abep.org/criterio-brasil (accessed November 2019).

25. Fischer JE, Bachmann LM \& Jaeschke R (2003) A readers' guide to the interpretation of diagnostic test properties: clinical example of sepsis. Intensive Care Med 29, 1043-1051.

26. Khanal V, Lee AH, Scott JA et al. (2016) Implications of methodological differences in measuring the rates of exclusive breastfeeding in Nepal: findings from literature review and cohort study. BMC Pregnancy Childbirth 16, 389. 